\title{
Management of severe aortic stenosis: the Singapore and Asian perspective
}

\author{
Edgar Lik Wui $\underline{T a y}^{1,2}$, MBBS, FACC, Jinghao Nicholas $\underline{\text { Ngiam }}^{3}$, MBBS, William KF $\underline{K o n g}^{1,2}$, MBChB, FRACP,

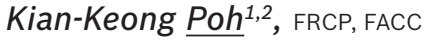

M anagement of aortic valve stenosis (AS) has seen a significant resurgence of interest in the past decade, as shown in the increasing number of publications on the topic and international valve conferences pertaining to valve-related diseases. This growth has been largely driven by the evolution of treatments available for this condition. While the options were previously largely surgical, the emergence of transcatheter aortic valve replacement (TAVR) has changed the landscape. ${ }^{(1)}$ In addition, closer collaboration among heart teams and imaging advances in echocardiography and computed tomography have contributed to the rapid change. The recently updated American College of Cardiology guidelines on the management of valvular heart disease reflected this. ${ }^{(2)}$ The pace of change in Asia, however, appears less dramatic compared to that in the United States and Europe. This article described the unique characteristics of the Asian cohort and their impact on the contemporary management of aortic stenosis.

\section{EPIDEMIOLOGY IN ASIA}

Asia is the largest continent in the world and consists of markedly heterogeneous ethnicities. The geographic differences in life expectancy rates (marked range of 62.2-83.7 years at birth) provide further insights on the varying aetiologic burden of AS across Asia, according to statistics from the World Health Organization. ${ }^{(3)}$ Given that the probability of degenerative AS only increases significantly after 75 years of age, some Asian patients do not have time to develop degenerative AS. In addition, childhood rheumatic fever rates also vary significantly among Asian countries. Currently, rheumatic aortic stenosis is most common in Asia, with a reported prevalence of 1.86 in China, 4.54 in India, and 1.3 in Bangladesh per 1,000 population. However, it is important to note that several Asian countries, including Singapore, mirror Western cohorts in having a very low prevalence of rheumatic valvular disease (e.g. 0.14 in Japan and 0.5 in South Korea per 1,000 population). This has significant implications, as rheumatic AS patients often present at a younger age and most would benefit from conventional open heart surgery. ${ }^{(4)}$ On the other hand, Singapore (similar to Japan and South Korea) has a high proportion of elderly with degenerative AS, and correspondingly, the uptake of TAVR is increasing.

\section{ANATOMIC DIFFERENCES}

Compared to Caucasian patients, most Asians with AS have a smaller body surface area, which affects the choice of therapy. Using computed tomography, we have demonstrated that the aortic annuli of Singaporean patients are smaller (unpublished study). Other studies have suggested that their iliofemoral arterial sizes are also smaller. ${ }^{(5)}$ While this may affect the TAVR approach, the availability of small French sizes (e.g. in the Evolut R systems) delivery systems allow for a transfemoral route when the femoral sizes are $5 \mathrm{~mm}$ and above. Nevertheless, in patients who have borderline femoral sizes with calcification and peripheral vascular disease, this remains important. Overall, the number of patients requiring the transapical approach has reduced significantly over the years, as observed in the Society of Thoracic Surgeons/ American College of Cardiology Transcatheter Valve Therapy Registry. ${ }^{(6)}$

The Japanese TAVR cohorts have a smaller aortic root annular diameter $(20.4 \pm 1.46 \mathrm{~mm}$ vs. $22.0 \pm 1.84 \mathrm{~mm} ; \mathrm{p}<0.01) .{ }^{(7)}$ This may result in more difficult transfemoral access patients for TAVR and may lead to the increased utilisation of alternative access sites. In contrast, for patients with very small annuli, surgeons may be reluctant to perform a root enlargement procedure and may prefer TAVR in some cases. Recent studies on bicuspid valves have also shed light on important interethnic differences. Asians tend to have Type I bicuspid valves with larger aortic root dimensions compared to Western cohorts. This information would have a significant impact on device sizing when the TAVR technology is expanded to younger patients, in whom a higher proportion of aortic stenosis cases have a bicuspid aetiology. ${ }^{(8)}$

Left ventricular outflow track (LVOT) annular size affects classification of AS as well. ${ }^{(9)}$ Michelena et al investigated whether LVOT diameters are a source of inconsistencies in the echocardiographic severity grading of AS based on transaortic gradients and aortic valve areas (AVAs). They divided patients into small, average and large LVOT diameters of $1.7-1.9 \mathrm{~cm}, 2.0-2.2 \mathrm{~cm}$ and $\geq 2.3 \mathrm{~cm}$. The study found that an AVA cutoff of $0.8 \mathrm{~cm}^{2}$ and $0.9 \mathrm{~cm}^{2}$, respectively, reduced severe AS categorisation inconsistency for patients with small LVOT diameter and average LVOT diameter. The current guideline's AVA cutoff of $1 \mathrm{~cm}^{2}$ was consistent for patients with large LVOT diameter. ${ }^{(9)}$ In our institution's retrospective database of 1,690 echocardiograms with isolated AS, $40 \%, 48 \%$ and $12 \%$ had LVOT diameters of $1.7-1.9 \mathrm{~cm}, 2.0-2.2 \mathrm{~cm}$ and $\geq 2.3 \mathrm{~cm}$, respectively. This differs from Michelena et al's series of $9 \%$, $56 \%$ and $35 \%$, respectively. ${ }^{(9)}$ For severe AS, our proportions were $52 \%, 31 \%$ and $17 \%$, respectively (from an unpublished study). This large proportion of local subjects with small LVOT diameters may imply that we should have a lower cutoff for

${ }^{1}$ Department of Cardiology, National University Heart Centre Singapore, National University Health System, ${ }^{2}$ NUS Yong Loo Lin School of Medicine, National University of Singapore, ${ }^{3}$ Department of Medicine, National University Health System, Singapore

Correspondence: A/Prof Kian-Keong Poh, Department of Cardiology, National University Heart Centre Singapore, National University Health System, 1E Kent Ridge Rd, NUHS Tower Block, Level 9, Singapore 119228. kian_keong_poh@nuhs.edu.sg 
classifying patients into the severe AS category. Another recent study also demonstrated a large number of bicuspid aortic valves among AS patients in China who were screened for TAVR with computed tomography, a significantly higher number than in other Asian studies published thus far. ${ }^{(10)}$ We postulate that the differences from Western cohorts may be related to the age of patients in the Chinese cohort compared to the other registries. This trend warrants further investigation, as it has implications for the treatment of AS patients with bicuspid valves. Currently, TAVR in this group remains off-label, as patients with severe bicuspid AS are associated with increased procedural complexity, and possibly inferior outcomes in the form of lower procedural success and an increase in periprocedural complications, although a published study did not demonstrate a significant difference in longer-term mortality. ${ }^{(11)}$ Several Chinese hospitals have independently treated many of these patients with success. ${ }^{(12)}$

The specific subgroup of paradoxical low-flow AS despite preserved left ventricular ejection fraction (LVEF) remained an important group that was challenging to manage. The proportions of low-flow severe AS (stroke volume index $<35 \mathrm{~mL} / \mathrm{m}^{2}$ ) with preserved LVEF in our series were similar to those of Western populations. This group of subjects appeared to have similar clinical outcomes compared to normal-flow AS in a paired echocardiographic analysis. ${ }^{(13)}$ We have also described that the paradoxical low-flow phenomenon was present even in moderate aortic stenosis. ${ }^{(14)}$ The outcomes of valve replacement (whether transcatheter or surgical) in this subgroup of patients in our Asian context have yet to be further elucidated.

\section{SURGICAL RISK SCORE AND FRAILTY}

Assessment and selection of AS patients for open surgery or TAVR is performed by a heart team consisting of cardiac surgeons and interventionists. In our institution, these cases were discussed in a valve forum led by non-invasive cardiologists with interest in valvular heart disease. In most instances, an integrative approach is used, which assesses comorbidities and utilises surgical risk scores (STS), clinical history and physical assessment, including assessment of frailty, before a decision on the best-suited therapy is made. While these risk scores were not validated for the Asian cohort, several studies have utilised the STS with good predictability. A recent objective clinical frailty scale used in a Japanese cohort showed good discriminability to exclude frail patients for whom invasive therapy would be futile. ${ }^{(15)}$

\section{THERAPY OPTIONS}

Open aortic valve surgery remains the most common treatment option for AS in Asia. Its key advantages are the long history and experience of cardiac surgeons in this region. Most of the studies available in the existing literature are retrospective and include patients with an age range of 70-80 years. The reported short-term outcomes are good and show no significant differences among types of prostheses.

The decision-making process regarding the choice of prosthesis (metallic vs. bioprosthetic) recommended by Asian surgeons differs slightly from that of their Western counterparts.
Utilising only the recommended age-related cutoff may not be applicable in many Asian sites. This is because life expectancy in different parts of Asia differs not only based on cardiac condition, systemic illnesses and gender, but also socioeconomic status and specific geographic location. The types of valves selected are also determined by cost, availability of the site to monitor the patient and ease of access to anticoagulation treatment and monitoring (apart from the patient's preference and life expectancy).

\section{THE FUTURE OF TAVR}

TAVR has been available in Asia for the past eight years, enabling many more patients to undertake treatment. Starting from Singapore, it is now available in many Asian cities, with Japan having the largest number of implants to date. Of note, a Singaporean study showed that a large proportion of patients declined open surgery despite being told of its benefits. ${ }^{(16)}$ This mirrors the mindset of patients in Asia, who often fear open surgery.

Despite this, the adoption of TAVR in Asia compared to the United States and Europe has been comparatively slow. The high cost of treatment remains a significant barrier to its uptake, largely due to the lack of full reimbursement for TAVR in most parts of Asia (the only exception being Japan). The high upfront cost of the implant remains prohibitive to many patients compared to traditional open heart surgery, which has much lower implant costs. Cost-effectiveness data from Singapore is unavailable. To remedy this, two projects are currently ongoing in our unit. The first, a value-driven outcomes analysis to determine the true cost of the TAVR procedure and the true value to the patient, would enable us to study opportunities to lower the cost of the procedure while maintaining optimal outcomes for patients. The second is a traditional cost-effectiveness analysis to determine if the cost of TAVR fulfils the institution's approved incremental cost-effectiveness ratio ranges, so that longer-term reimbursement becomes possible. Further challenges in the uptake of TAVR in Singapore and Asia include possible under-diagnosis of AS in the Asian population. Lack of awareness also remains a challenge, as many patients, even those diagnosed with severe AS, still do not know that TAVR is available as a treatment option.

Despite these challenges, the outcomes of TAVR for AS patients remain promising. ${ }^{(17,18)}$ Regionally, several innovative projects in transcatheter heart valve development are being undertaken, and may improve the availability of Asian-made valves. For example, the Venus A-valve from China is now commercially available in mainland China, with the MicroPort valve being evaluated as well. In India, the Myvalve is being utilised in a clinical trial, while Thailand has developed the Hydra valve in parallel. ${ }^{(19)}$ These innovations may further fuel the growth of TAVR.

Singapore is fortunate to have early access to therapies via several special access routes, as well as the availability of most commercialised transcatheter valves. In addition, both the Asian TAVR registry ${ }^{(20)}$ and the Japanese OCEAN-TAVI (Optimized Transcatheter Valvular Intervention) registry ${ }^{(21)}$ have published clinical outcomes demonstrating success in patient selection and the safety of the therapy. As with the Western cohorts, there 
is a gradual shift in which TAVR is increasingly being offered to patients of intermediate and even lower surgical risk, although cost remains an important consideration. It is anticipated that the utilisation of TAVR in Asia will continue to expand as more patients learn about this treatment.

\section{REFERENCES}

1. Leon MB, Smith CR, Mack M, et al; PARTNER Trial Investigators. Transcatheter aortic-valve implantation for aortic stenosis in patients who cannot undergo surgery. N Engl J Med 2010; 363:1597-607.

2. Nishimura RA, Otto CM, Bonow RO, et al. 2017 AHA/ACC focused update of the 2014 AHA/ACC guideline for the management of patients with valvular hear disease: a report of the American College of Cardiology/American Hear Association Task Force on Clinical Practice Guidelines. J Am Coll Cardiol 2017; 70: 252-89.

3. World Health Organization. Global health observatory data. Available at: http:// www.who.int/gho/en/. Accessed March 25, 2018

4. Nobuyoshi M, Arita T, Shirai S, et al. Percutaneous balloon mitral valvuloplasty: a review. Circulation 2009; 119:e211-9.

5. Chiam PT, Koh AS, Ewe SH, et al. Iliofemoral anatomy among Asians: implications for transcatheter aortic valve implantation. Int J Cardiol 2013; 167:1373-9.

6. Thourani $\mathrm{VH}$, Jensen $\mathrm{HA}$, Babaliaros $\mathrm{V}$, et al. Transapical and transaortic transcatheter aortic valve replacement in the United States. Ann Thorac Surg 2015; 100:1718-27.

7. Watanabe Y, Hayashida K, Takayama M, et al. First direct comparison of clinical outcomes between European and Asian cohorts in transcatheter aortic valve implantation: the Massy study group vs. the PREVAIL JAPAN trial. J Cardio 2015; 65:112-6

8. Kong WKF, Regeer MV, Poh KK, et al. Inter-ethnic differences in valve morphology, valvular dysfunction and aortopathy between Asian and European patients with bicuspid aortic valve. Eur Heart J 2018; 39:1308-13.

9. Michelena HI, Margaryan E, Miller FA, et al. Inconsistent echocardiographic grading of aortic stenosis: is the left ventricular outflow tract important? Heart 2013; 99:921-31.
10. Jilaihawi $\mathrm{H}, \mathrm{Wu} \mathrm{Y,} \mathrm{Yang} \mathrm{Y}$, et al. Morphological characteristics of severe aortic stenosis in China: imaging corelab observations from the first Chinese transcatheter aortic valve trial. Catheter Cardiovasc Interv 2015; 85 Suppl 1:752-61

11. Yoon $\mathrm{SH}$, Bleiziffer $\mathrm{S}$, De Backer $\mathrm{O}$, et al. Outcomes in transcatheter aortic valve replacement for bicuspid versus tricuspid aortic valve stenosis. J Am Coll Cardiol 2017; 69:2579-89.

12. Perlman GY, Blanke $P$, Webb JG. Transcatheter aortic valve implantation in bicuspid aortic valve stenosis. Eurointervention 2016; 12:Y42-5.

13. Ngiam JN, Tan BY, Sia CH, et al. Comparing characteristics and clinical and echocardiographic outcomes in low-flow vs normal-flow severe aortic stenosis with preserved ejection fraction in an Asian population. Echocardiography 2017; 34:638-48

14. Tan YQ, Ngiam JN, Kong WK, Yeo TC, Poh KK. Prevalence, clinical and echocardiographic characteristics of various flow and gradient patterns in mild or moderate aortic stenosis with normal left ventricular ejection fraction. Int J Cardiol 2016; 221:1107-15.

15. Shimura T, Yamamoto $M$, Kano $S$, et al. Impact of the clinical frailty scale on outcomes after transcatheter aortic valve replacement. Circulation 2017; 135:2013-24.

16. Tay EL, Lew PS, Poh KK, et al. Demographics of severe valvular aortic stenosis in Singapore. Singapore Med J 2013; 54:36-9.

17. Chew N, Hon JK, Yip WL, et al. Mid-term study of transcatheter aortic valve implantation in an Asian population with severe aortic stenosis: two-year Valve Academic Research Consortium-2 outcomes. Singapore Med J 2017; 58:543-550.

18. Tay EL, Poh KK, Yip JW, Kong WK. Transcatheter aortic valve implantation: from revolution to evolution. Singapore Med J 2016; 57:406-7.

19. Wiegerinck EM, Van Kesteren F, Van Mourik MS, Vis MM, Baan J Jr. An up-to-date overview of the most recent transcatheter implantable aortic valve prostheses. Expert Rev Med Devices 2016; 13:31-45.

20. Yoon SH, Ahn JM, Hayashida K, et al. Clinical outcomes following transcatheter aortic valve replacement in Asian population. JACC CardiovasC Interv 2016; 9:926-33.

21. Yashima F, Yamamoto $M$, Tanaka $M$, et al. Transcatheter aortic valve implantation in patients with an extremely small native aortic annulus: the OCEAN-TAVI registry. Int J Cardiol 2017; 240:126-31. 